\title{
MALIGNANT MELANOMA OF THE ORBIT IN A CASE OF OCULODERMAL MELANOSIS* (NAEVUS OF OTA)
}

\author{
BY \\ BARRIE JAY \\ Department of Pathology, Institute of Ophthalmology, University of London
}

APART from the value of recording a further example of a rare disease, it is hoped that this case of a malignant melanoma of the orbit occurring in a patient with oculodermal melanosis will shed some light upon certain aspects of melanotic lesions.

\section{Case Report}

A 64-year-old woman was admitted to the Newcastle upon Tyne General Hospital (under the care of Mr. Fenton Braithwaite) with a one year's history of progressive protrusion of her right eye. She had pigmentation of the skin of her right lower lid and temporal region, melanosis bulbi, and unilateral proptosis resulting in restriction of ocular movements. The right orbit was decompressed and a biopsy of orbital tissue showed fat and fibrous tissue only. She was re-admitted to hospital six months later with a recurrence of the right proptosis and a soft tumour was felt in the right temporal region, the lateral wall of the orbit having been removed at the previous operation. In view of these findings the right orbital contents were removed en bloc with the temporalis muscle and the pigmented area of temporal skin. Ten months later the patient died from congestive cardiac failure and multiple secondary deposits of melanoma. It is not known whether a postmortem examination was performed.

\section{Pathological Findings}

Macroscopic Examination.-The specimen consisted of the right orbital contents and eyelids, together with a large flap of temporal skin and underlying temporalis muscle. A partially pigmented retro-ocular tumour was present, closely applied to the posterior pole of the eye and located mainly above the optic nerve. Several poorly defined areas of pigmentation were present beneath the bulbar and palpebral conjunctiva and a few oval pigmented lesions were present in the substance of the lower lid.

Microscopic Examination.-The specimen showed the pathological features of two distinct, though probably related, conditions:

(1) Malignant melanoma of the orbit. The retro-ocular tumour was found to be a lightly pigmented malignant melanoma composed predominantly of anaplastic 
epithelioid cells lying in a well-marked reticulin framework. Areas of necrosis and haemorrhage were present in the tumour which was partly enclosed in a fibrous capsule (Figs 1 and 2).

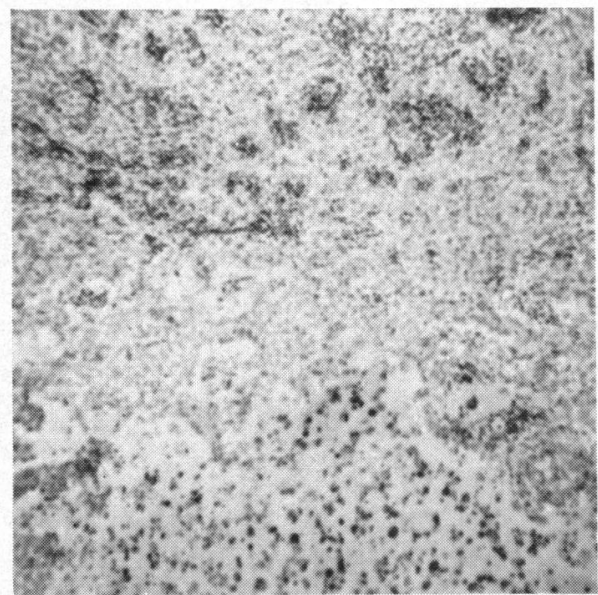

Fig. 1.-Malignant melanoma of the orbit containing areas of necrosis and haemorrhage. H. \& E. $\times 90$.

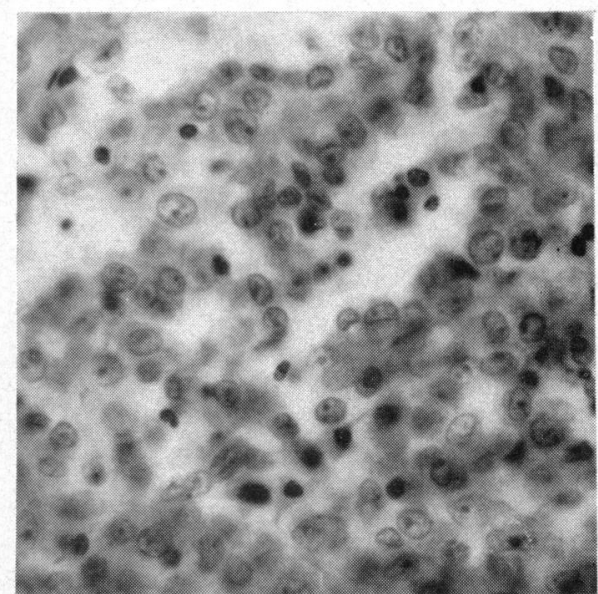

FIG. 2.-High-power view of tumour cells shown in Fig. $1 . \quad \times 415$.

(2) Oculodermal melanosis. There was marked melanosis of the whole uveal tract resulting from an increased number of apparently normal fusiform melanocytes in this tissue (Fig. 3). Similar cells were present along the intra-scleral channels (Fig. 4) and in the optic nerve-head, but there was no evidence of an intra-ocular tumour. Scattered fusiform melanocytes were present in the dermis of the eyelids (Figs 5 and 6) and temporal skin. These cells lay parallel to the surface of the skin and did not disrupt its architecture. Similar cells were present in the orbicularis and temporalis muscles and in the orbital tissue. Small circumscribed heavily pigmented tumours

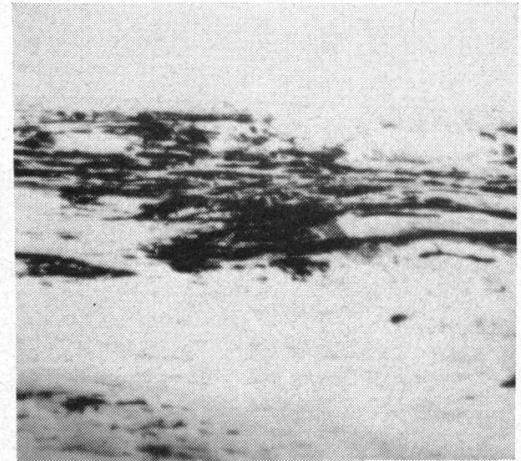

FIG. 3.--Melanosis of the choroid resulting from an increased number of fusiform melanocytes in this tissue (the retina separated from the choroid during fixation and is not present in this field). H. \& E. $\times 90$.

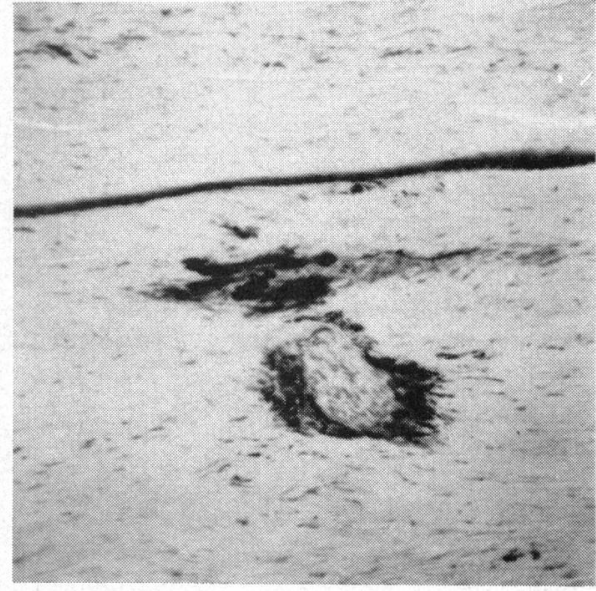

Fig. 4.-Fusiform melanocytes present along intrascleral channels. H. \& E. $\times 90$. 


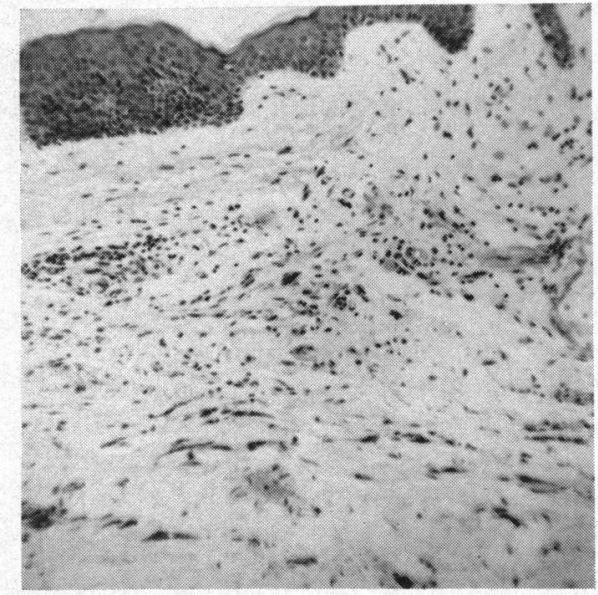

FIG. 5.-Dermal melanosis of the eyelid. Melanocytes lying parallel to the surface of the skin are present deep in the dermis and do not disrupt its architecture. H. \& E. $\times 90$.

were present in the substance of the eyelids (Fig. 7); they were composed of densely packed fusiform cells of benign appearance similar to the scattered cells present elsewhere in the specimen.

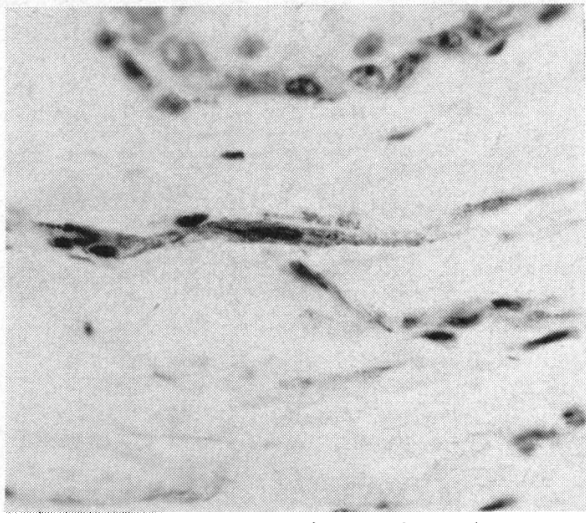

FIG. 6.-High-power view of melanocytes shown in Fig. $5 . \quad \times 415$.

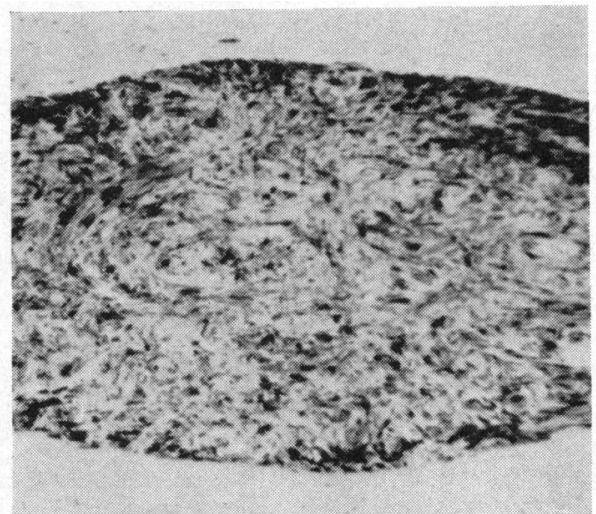

Fig. 7.-Blue naevus of eyelid composed of heavily pigmented fusiform melanocytes. H. \& E. $\times 90$.

\section{Discussion}

Besides developing a malignant melanoma of the orbit, this patient had a widespread disturbance of melanin pigmentation involving the tissues over the distribution of the ophthalmic and maxillary divisions of the trigeminal nerve. This widespread melanotic lesion consisted of spindle-shaped melanocytes situated in the skin, orbicularis muscle, conjunctiva, sclera, uveal tract, and orbital tissue, these melanocytes being identical in appearance with those that occur in Mongolian spots and blue naevi. In Mongolian spots and dermal melanoses the aberrant melanocytes are sparsely scattered in the dermis, while in blue naevi they are present in greater numbers, forming a tumour and disrupting the normal architecture of the skin. The association of melanosis bulbi with a dermal melanosis over the distribution of the trigeminal nerve is known eponymously as the naevus of Ota, or naevus fuscocaeruleus ophthalmo-maxillaris (Ota, 1939). The first description of this condition was that by Hulke (1861) and since then there have been scattered reports of similar cases in the literature (Mishima and Mevorah, 1961). Lund and Kraus (1962) considered the naevus of Ota to be a dermal melanosis rather than a tumour, while Ito 
(1952) believed it to be a form of blue naevus. Dorsey and Montgomery (1954) described the naevus of Ota as essentially a Mongolian spot located on the side of the face and involving the eye, its histology varying between that of a Mongolian spot and that of a cellular blue naevus. It seems probable that melanosis bulbi and the naevus of Ota are different degrees of severity of the same abnormality, and in order to emphasize both the occasional association between dermal melanosis and melanosis bulbi, and the relationship they bear to each other, it is suggested that the term "oculodermal melanosis" (Lund and Kraus, 1962) be applied to the naevus of Ota. The histological appearance of the present case supports the view of Dorsey and Montgomery (1954), for it is essentially a dermal melanosis with small scattered blue naevi in the eyelids.

Tanino (1939) described 26 cases of oculodermal melanosis and classified them into five types:

(1) Mild orbital type. Faint pigmented spots scattered over the upper and lower eyelids and in the skin over the temple.

(2) Mild zygomatic type. Pigmentation in the skin between the sulcus infrapalpebralis and sulcus nasolabialis.

(3) Moderate type. Pigmentation in the skin of the eyelids, zygomatic region, cheek, and temple.

(4) Intensive type. The pigmentation extends further, involving the scalp, forehead, and brow.

(5) Bilateral type.

In Tanino's (1939) series the eye was involved in 17 cases. In the series of cases reported by Ito (1952), 65 were present at birth and 41 appeared after birth. This condition of oculodermal melanosis, which is most frequently seen in oriental races, has been reported in Caucasians (Dorsey and Montgomery, 1954) and in Negroes (Mishima and Mevorah, 1961).

In order to explain the occurrence of aberrant melanocytes in tissues over the distribution of the trigeminal nerve it is necessary to postulate either that the ultimate distribution of these pigment-producing cells is determined by the presence of nerves, or that these cells arise from constituents of the nerves. There is considerable experimental evidence that melanoblasts migrate from the neural crest in embryo and differentiate into melanocytes when they reach their destination in skin, mucous membrane, uveal tract, and leptomeninges. This has been shown to be true for amphibia, birds, and mammals (Rawles, 1947), although experimental confirmation for its occurrence in man is still lacking. There is evidence, however, that in the first half of human foetal development melanoblasts are present in a fairly definite layer in the dermis, and that by the eleventh or twelfth week of foetal life they have started to penetrate the epidermis (Zimmermann and Becker, 1959). Mongolian spots and dermal melanoses represent postnatal remnants of this general pigmentary layer in the dermis, a layer which occurs normally in the dermis of adult apes (Adachi, 1903). In the present case the occurrence of melanocytes along the intrascleral channels is evidence of their close association with intrascleral nerves, and as both melanoblasts and Schwann cells are derived from the neural crest, these cells may arise from common precursors which differentiate when they reach their destination, or they may arise separately yet reach their destination by the same pathway. 
Although it is generally agreed that dermal melanocytes originate from the neural crest and are atavistic remnants of the dermal pigmentary layer, no one has yet answered the question posed by Dorsey and Montgomery (1954) as to why, if blue naevus cells and ordinary naevus cells are of the same origin, the ordinary naevus cells do not show dendritic processes-like the blue naevus cells-when they drop down from the epidermis into the dermis. It is possible that the tumour-forming potentialities of melanocytes, once they have reached the basal layer of the epidermis, are affected by their proximity to epithelial cells, and this would also account for the rarity of malignant blue naevi as compared with malignant melanomata of the skin.

Malignant change has not been reported in a Mongolian spot or in a case of dermal melanosis, and in only three cases has a malignant melanoma arisen in oculodermal melanosis. In two of these cases (Dorsey and Montgomery, 1954) the malignant melanomata were considered to have arisen from subcutaneous blue naevi, while in a third case (Hulke, 1861) a malignant melanoma of the choroid developed. In contrast to this rare occurrence of malignant change in oculodermal melanosis, malignant melanomata of the uveal tract develop in cases of melanosis bulbi more frequently than can be expected by chance (Duke-Elder, 1938).

The association of two rare conditions, malignant melanoma of the orbit and oculodermal melanosis, as in the present case, is unlikely to occur by chance and, since oculodermal melanosis may present only faint pigmentation of the skin, one wonders whether it has been missed in previously reported cases of primary malignant melanoma of the orbit. This association of melanosis bulbi with dermal melanosis also suggests that these two conditions may have a similar aetiology, and if this is the case a "naevus" of the uveal tract could be equated to a blue naevus of the skin, and a malignant melanoma of the uveal tract to a malignant blue naevus of the skin. This view is supported by Ashton (1964), who stated that naevus cells have never been conclusively demonstrated in the uveal tract, nor could they be expected to occur at this site, for they are a strain of cells arising from epidermal melanocytes.

\section{Summary}

A case is presented of a malignant melanoma of the orbit arising in association with oculodermal melanosis. The rare association of melanosis bulbi with dermal melanosis suggests that these two conditions have a similar aetiology and the implications of this hypothesis are discussed.

I should like to thank Mr. Fenton Braithwaite for permission to publish the clinical details of this case, and Professor Norman Ashton for his unfailing encouragement and advice.

\section{REFERENCES}

AdACHI, B. (1903). Z. Morph. Anthrop., 6, 1.

AsHTON, N. (1964). Brit. J. Ophthal., 48, 650.

DORSEY, C. S., and MONTGOMERY, H. (1954). J. invest. Derm., 22, 225.

DUKE-ElDER, W. S. (1938). " "Text-book of Ophthalmology", vol. 2, p. 1395 . Kimpton, London.

HulKe, J. W. (1861). Ophthal. Hosp. Rep., 3, 279.

Iтo, M. (1952). Tohoku J. exp. Med., 55, suppl. 1, p. 21.

LuND, H. Z., and KraUS, J. M. (1962). "Atlas of Tumor Pathology", sect. 1, fasc. 3: Melanotic Tumors of the Skin, p. 25. Armed Forces Institute of Pathology, Washington.

Mishima, Y., and Mevorah, B. (1961). J. invest. Derm., 36, 133.

OtA, M. (1939). Jap. J. Derm., 46, 99.

Rawles, M. E. (1947). Physiol. Zool., 20, 248.

Tanino, H. (1939). Jap. J. Derm., 46, 107.

ZimmermanN, A. A., and Becker, S. W. Jr. (1959). In "Pigment Cell Biology", ed. M. Gordon, p. 159. Academic Press, New York. 